\title{
Interdevice agreement in respiratory resistance values by oscillometry in asthmatic children
}

\author{
Francine M. Ducharme (10 ${ }^{1,2,3}$, Imane Jroundi ${ }^{3,4}$, Guillaume Jean ${ }^{3}$, \\ Guillaume Lavoie Boutin ${ }^{3}$, Christiane Lawson ${ }^{3}$ and Benjamin Vinet ${ }^{3}$
}

Affiliations: ${ }^{1}$ Dept of Pediatrics, University of Montreal, Montreal, QC, Canada. ${ }^{2}$ Dept of Social and Preventive Medicine, University of Montreal, Montreal, QC, Canada. ${ }^{3}$ Clinical Research on Childhood Asthma Research Unit, Research Centre, Sainte-Justine University Health Centre, Montreal, QC, Canada. ${ }^{4}$ Unit of Training and Research in Public Health, School of Medicine and Pharmacy of Rabat, University of Mohamed V, Rabat, Morocco.

Correspondence: Francine M. Ducharme, Dept of Pediatrics, CHU Hospital Sainte-Justine, Room B.17.000, 3175 Chemin de la Côte-Sainte-Catherine, Montreal, QC H3T 1C5, Canada.

E-mail: francine.m.ducharmedumontreal.ca

\section{ABSTRACT}

Background: With several commercially available devices measuring respiratory impedance by oscillometry, the agreement between values obtained on different instruments or frequencies remains unclear. Our aim was to examine the agreement between resistance and reactance parameters on two oscillometry instruments using different waveforms.

Methods: We conducted a prospective cross-sectional study in asthmatic children aged 3-17 years. Reproducible oscillometry measurements were obtained in random order, by blinded operators, at three modes: 5-10-15-20-25 Hz (5-25 Hz) multifrequency mode on the MasterScreen impulse oscillometry system, and both 5-25 Hz multifrequency mode and $7 \mathrm{~Hz}$ monofrequency on the tremoFlo C-100 airwave sinusoidal system. Resistance, reactance and within-breath parameters were examined using the intraclass correlation coefficient (ICC), paired t-test, linear regression and Bland-Altman method.

Results: Of 50 participants, 44 and 38 completed between-device and within-frequency measurements, respectively. Between-device measurements at $5-25 \mathrm{~Hz}$ showed high (ICC 0.88-0.91) and good (ICC 0.69-0.87) agreement in resistance and reactance, respectively, but with an absolute within-patient difference $\left(\geqslant 0.05 \mathrm{kPa} \cdot \mathrm{L}^{-1} \cdot \mathrm{s}^{-1}\right)$ and proportional bias $\left(\geqslant 30 \%\right.$ per $\left.\mathrm{kPa} \cdot \mathrm{L}^{-1} \cdot \mathrm{s}^{-1}\right)$ in all parameters and oscillatory frequencies, apart from resistance at $5 \mathrm{~Hz}$. A significant proportional bias was documented in most within-breath parameters at 5 versus $7 \mathrm{~Hz}$ on tremoFlo.

Conclusion: Observed differences in resistance and reactance suggest the need for instrument- and frequency-specific paediatric normative values.

@ERSpublications

Agreement between forced oscillation values using impulse versus airwave sinusoidal oscillations in children is excellent for resistance at $5 \mathrm{~Hz}$ but a proportional bias exists in resistance at $\geqslant 10 \mathrm{~Hz}$ and reactance, calling for better device harmonisation http://ow.ly/DlNc30nrM5g

Cite this article as: Ducharme FM, Jroundi I, Jean G, et al. Interdevice agreement in respiratory resistance values by oscillometry in asthmatic children. ERJ Open Res 2019; 5: 00138-2018 [https:// doi.org/10.1183/23120541.00138-2018].

This article has supplementary material available from openres.ersjournals.com.

Received: July 172018 | Accepted after revision: Jan 072019

Copyright $\odot$ ERS 2019. This article is open access and distributed under the terms of the Creative Commons Attribution Non-Commercial Licence 4.0. 


\section{Introduction}

Objective assessment of respiratory function is crucial for the diagnosis and monitoring of patients with asthma [1]. Whereas spirometry remains the gold standard, oscillometry (or forced oscillation technique) is one of several techniques to measure lung function that has been shown to be discriminative and responsive to change [2-5]. Importantly, oscillometry is an effort-independent technique requiring minimal cooperation, which makes it very attractive in children, particularly in pre-schoolers and those too sick to cooperate with spirometry [6]. The MasterScreen (CareFusion, Hochberg, Germany) uses an impulse oscillometry system (IOS) producing a square wave (more accurately, a Dirac function) to generate oscillations. Several recently commercialised instruments, such as the tremoFlo C-100 (Thorasys, Montreal, QC, Canada), MostGraph (CHEST M.I., Tokyo, Japan) and Resmon Pro (Medical Graphics Diagnostics, Rome, Italy), generate multifrequency oscillations using gentle sinusoidal airwaves superimposed on the patient's respiratory airflow. The use of different oscillation technologies, transducers and analytic algorithms raises the question as to whether the agreement between values obtained on different oscillometry instruments is sufficient to use the same normative and interpretative cut-off values, interchangeably.

Commercially available since 1992, most published reference values have been developed on the IOS in Caucasian [7-10] and non-Caucasian children [11-13]; to the best our knowledge, only one has been published on the tremoFlo, in Emirati children [14]. Yet, using fixed phantom models, the MasterScreen IOS and MostGraph did not generate identical impedance values, with up to $10 \%$ variation from the test charges (i.e. a resistor with known resistance), resulting in differences in resistance and reactance [15]. Whereas similar resistance values were observed in healthy adults between the tremoFlo, Resmon Pro and a custom-built device ("WIMR"), $>10 \%$ variation was observed when compared with the IOS [16]. The tremoFlo offers a monofrequency mode at $7 \mathrm{~Hz}$, which may be preferred to $5 \mathrm{~Hz}$ to improve the signal/ noise ratio in children with a higher respiratory rate [17]. Clinical interpretation of values at $7 \mathrm{~Hz}$ is hampered by the absence of paediatric reference values at this frequency; perhaps using $5 \mathrm{~Hz}$ reference values with a correction factor could address the issue. To the best of our knowledge, no between-device comparison of parameters measured at 5 versus $7 \mathrm{~Hz}$ has been published in children.

The objectives of this study were to examine, in asthmatic children, the agreement between 1) various respiratory impedance parameters at $5-25 \mathrm{~Hz}$ on the MasterScreen IOS and the tremoFlo C-100, and 2) total- and within-breath parameters measured at 5 versus $7 \mathrm{~Hz}$ on the tremoFlo.

\section{Methods}

We conducted a prospective cross-sectional study, with three sets of oscillometry measurements, in children presenting to the Asthma Clinic of the Sainte-Justine University Hospital Centre (Montreal, QC, Canada), a tertiary care academic centre. The study was approved by the Institutional Review Board. Parents provided written informed consent and assent was obtained in children aged $\geqslant 8$ years.

Children were eligible if they fulfilled the following conditions: 1) aged 3-17 years, 2) suspected or confirmed asthma, 3) no intake of short-acting bronchodilators in the $4 \mathrm{~h}$ preceding measurements, 4) no spirometry testing in the $30 \mathrm{~min}$ preceding measurements, 5) ability to collaborate with oscillometry, and 6) prior consent to participate in our Paediatric Asthma Database and Biobank. Children were excluded if they were not able to breathe through a mouthpiece without a leak or if enrolled in another study.

We documented basic demographics including age, sex, ethnicity, height and weight. Asthma control was ascertained using the validated Childhood Asthma Control Test (cACT) [18] for children aged 4-11 years and the Asthma Control Test (ACT) [19] for children aged $\geqslant 12$ years; a value $<20$ on either test was indicative of poor asthma control. Measurements of respiratory impedance were obtained in random order at the 5-10-15-20-25 Hz multifrequency mode (hereafter referred to as 5-25 Hz) on the MasterScreen IOS and the tremoFlo C-100 using the harmonic soft impulse signal, and at the $7 \mathrm{~Hz}$ monofrequency on the tremoFlo. The order of the three test modes was determined by a simple randomisation procedure with a fixed block size of 6; allocation concealment was achieved by consecutively numbered opaque envelopes containing the test order. Oscillometry measurements were obtained using a previously described standardised technique [2]. Briefly, children breathed quietly through the mouthpiece wearing a nose clip, with the chin and neck positioned at $90^{\circ}$, and with the technician, parents or patient holding the child's cheeks to minimise the upper airway shunt. At least four consecutive breathing cycles, without any evidence of swallowing, obstruction of the mouthpiece with the tongue or other artefacts, were selected by the operator on the IOS, whereas a similar selection was made automatically, without the operator's influence, on the $16 \mathrm{~s}$ tremoFlo measurement. Two different operators, blinded to the results on the alternate oscillometry instrument, administered the tests on the tremoFlo and IOS, to ensure that the results of the tremoFlo did not influence the selection of breaths on the IOS. Up to six trials were performed for each measurement to obtain at least three resistance values at $5 \mathrm{~Hz}(R 5)$ or $7 \mathrm{~Hz}(R 7)$ with a 
coefficient of variation of $\leqslant 15 \%$, as criteria for reproducibility [2]. The average value of three reproducible measurements was reported for each parameter. The devices were calibrated daily according to the manual of procedures established from the user manuals.

\section{Statistics}

Assuming a high agreement, a sample of 45 patients was sufficient to identify an intraclass correlation coefficient (ICC) of 0.925 with a precision of \pm 0.075 , resulting in a $95 \%$ confidence interval encompassing 0.85-1.00 absolute agreement. Anticipating 10\% withdrawals due to insufficient reproducibility, we sought a priori to recruit 50 patients.

Categorical variables were reported as count (percentage), and continuous variables as mean with standard deviation and median with interquartile range. The absolute agreement between the within-patient average resistance $(R)$ and reactance $(X)$ values at each frequency $(5-25 \mathrm{~Hz})$ as well as the frequency dependence $\left(R_{5-20}\right.$ and $\left.X 5-20\right)$ on both devices was examined using the ICC: values $>0.90$ were indicative of excellent agreement, whereas values between $0.75-0.90$ and $0.50-0.75$ portrayed good and moderate agreement, respectively [20]. The possibility of two types of systematic bias was examined: a fixed bias was explored by the absolute within-patient difference in $\mathrm{kPa} \cdot \mathrm{L}^{-1} \cdot \mathrm{s}^{-1}$ (paired $\mathrm{t}$-test after confirming the normality of the distribution) and reported with $95 \%$ confidence intervals, whereas a proportional bias, i.e. an increasing (or decreasing) systematic deviation with increasing impedance, was examined by a linear regression model. Bland-Altman plots served to determine agreement and bias [21]. The same approaches were used to compare within-breath parameters obtained at 5 versus $7 \mathrm{~Hz}$ on the tremoFlo. Statistical tests were considered significant when $\mathrm{p} \leqslant 0.05$. No adjustment was made for multiple testing in this exploratory study. The data were analysed using SAS version 9.3 (SAS Institute, Cary, NC, USA) and SPSS Statistics for Windows version 24.0 (IBM, Armonk, NY, USA).

\section{Results}

Between January and May 2017, 137 patients were approached; 47 children were ineligible. Of the 90 eligible children, 50 (56\%) participants were randomised. Six patients did not complete all three testing modes. 46 patients completed testing at $5-25 \mathrm{~Hz}$ on both devices, with reproducible results documented in $38(83 \%)$ patients who contributed data to the tremoFlo-IOS agreement analysis. 44 children completed reproducible measurements at $5-25$ and $7 \mathrm{~Hz}$ on the tremoFlo, contributing data to the 5 versus $7 \mathrm{~Hz}$ agreement analysis (figure 1).

Among the 46 patients contributing data to any comparison, the mean \pm sD age of participants was $8.5 \pm 3.7$ years with $39 \%$ aged 3-6 years and there was a balanced sex distribution; $25 \%$ had poor clinical control and the mean \pm SD $R 5$ was $0.5 \pm 1.06$ with $15.8 \%$ displaying a $R 5$ z-score of $\geqslant 2$ (table 1) [9]. The mean coefficient of variation of $R 5$ was $8 \%$; the same was observed for impedance at 5-25 Hz (supplementary table E1).

Overall, the mean resistance values were slightly higher and the mean reactance slightly more negative on the tremoFlo than the IOS; the same held true when medians were considered. The ICC between the IOS and tremoFlo values at each oscillatory frequency $(5-25 \mathrm{~Hz})$ varied between 0.88 and 0.91 for resistance, and between 0.69 and 0.87 for reactance; it was 0.83 for $\Delta R 5-20,0.70$ for $\Delta X_{5-20}$ and 0.63 for resonant frequency (table 2).

As for fixed biases, there was no statistically significant absolute mean difference in resistance at most frequencies, with the exception of $10 \mathrm{~Hz}(\mathrm{p}=0.003)$ and $R 5-20(\mathrm{p}=0.04)$ where the absolute difference reached $\geqslant 0.05 \mathrm{kPa} \cdot \mathrm{L}^{-1} \cdot \mathrm{s}^{-1}$. However, the opposite was observed in reactance, where the absolute differences were statistically significant and exceeded $0.05 \mathrm{kPa} \cdot \mathrm{L}^{-1} \cdot \mathrm{s}^{-1}$ at all frequencies, except $10 \mathrm{~Hz}$. A statistically significant proportional difference was observed for all resistance and reactance parameters (except R5) where the tremoFlo and IOS values differed by a multiplicative factor varying between $0.30-0.42$ and $0.35-0.79$ for every $\mathrm{kPa} \cdot \mathrm{L}^{-1} \cdot \mathrm{s}^{-1}$ unit of resistance and reactance, respectively. A post hoc sensitivity analysis excluding an outlier present only at $R_{5}$ showed no evidence of a fixed bias (mean difference $0.00,95 \%$ CI $-0.05-0.06 ; p=0.95)$, but suggested a significant proportional bias $(\beta 0.27,95 \%$ CI 0.11-0.44; $\mathrm{p}=0.002)$. As graphically displayed in the Bland-Altman plots, resistance values were proportionally higher and reactance values more negative on the tremoFlo compared with the IOS, with increasing impedance (figure 2). The potential impact of the observed proportional difference on clinical interpretation was examined using the same IOS-specific normative values for both devices [9]. For any resistance of $0.75-1.5 \mathrm{kPa} \cdot \mathrm{L}^{-1} \cdot \mathrm{s}^{-1}$ on the IOS, the projected $R 10$ values measured on the tremoFlo resulted in a $\mathrm{z}$-score difference varying between 0.4 and 1.7 for both a typical 4.0 -year-old $(105 \mathrm{~cm})$ male and a typical 8.5-year-old $(129 \mathrm{~cm})$ male, with higher z-score attributed to the tremoFlo values (table 3 ).

The ICC between whole-breath, average inspiratory and expiratory parameters measured at $5 \mathrm{~Hz}$ on the $5-25 \mathrm{~Hz}$ multifrequency mode and $7 \mathrm{~Hz}$ monofrequency on the tremoFlo was $\geqslant 0.97$ for resistance and 


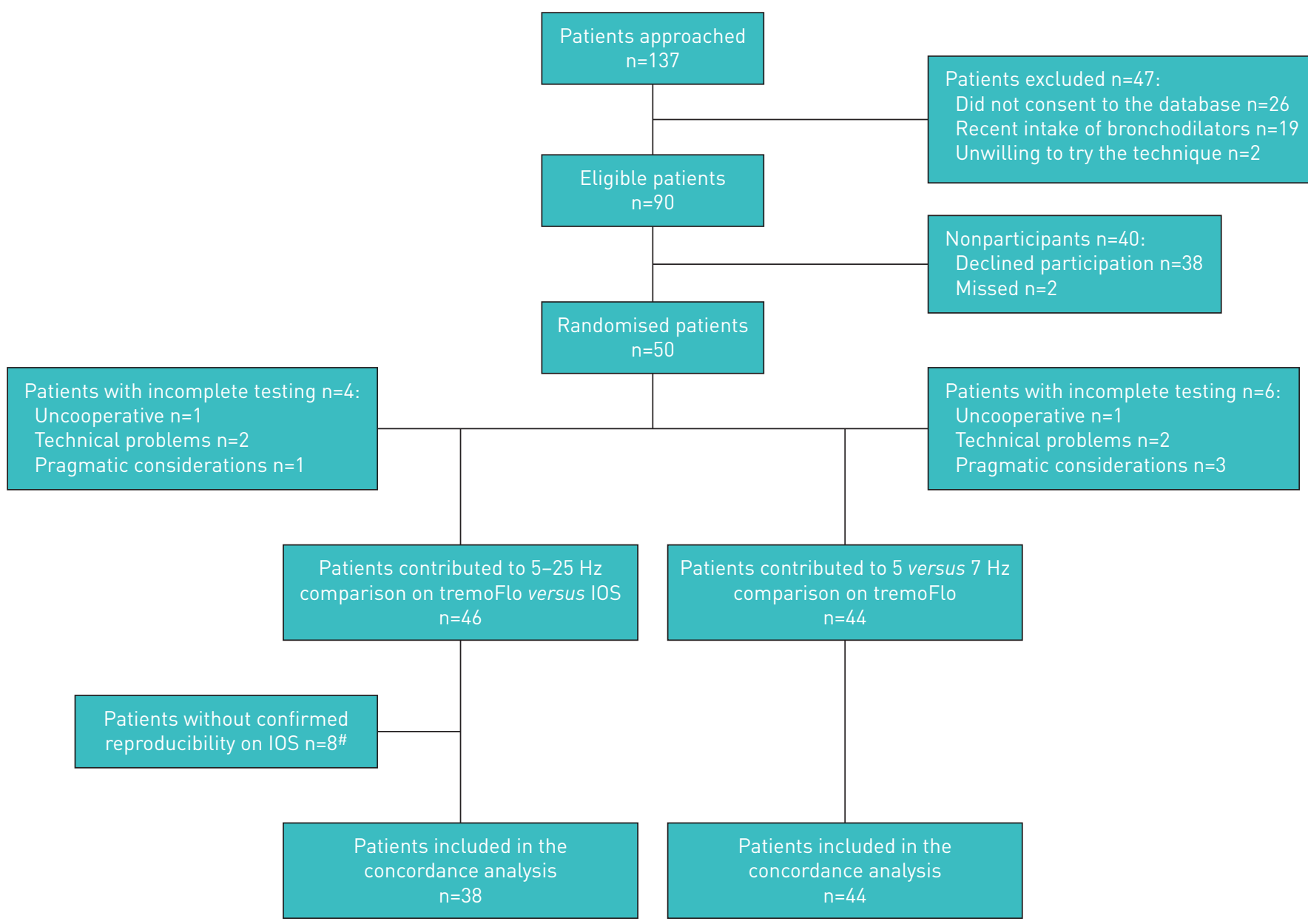

FIGURE 1 Patient selection from screening to analysis. IOS: MasterScreen impulse oscillometry system. ${ }^{\#}$ : in seven patients, the coefficient of variation of repeated measures exceeded $15 \%$, whereas in one patient, the mean of three values was recorded, but the individual values were lost, preventing confirmation of the coefficient of variation.

$\geqslant 0.94$ for both reactance and the within-breath difference of resistance $(\Delta R)$; it fell to 0.79 in within-breath reactance $(\Delta X)$. The absolute mean difference was statistically significant for inspiratory resistance $(\mathrm{p}=0.008)$ and for all reactance parameters including $\Delta X$, where it was $\geqslant 0.04 \mathrm{kPa} \cdot \mathrm{L}^{-1} \cdot \mathrm{s}^{-1}$ (supplementary table E2). There was a statistically significant proportional difference for resistance and reactance in all parameters, except inspiratory reactance; larger resistance and more negative reactance values were observed at 5 than $7 \mathrm{~Hz}$ (figure 3).

\section{Discussion}

In our cohort of asthmatic children, the within-patient absolute agreement (ICC) between the IOS and tremoFlo was excellent for resistance values and moderate-to-good for reactance. However, resistance measurements on the tremoFlo were consistently higher, reactance values more negative and resonance frequency higher than measured on the IOS. While the average within-patient absolute differences were generally clinically small $\left(<0.10 \mathrm{kPa} \cdot \mathrm{L}^{-1} \cdot \mathrm{s}^{-1}\right)$, a proportional bias was observed in resistance at $10-25 \mathrm{~Hz}$ and in reactance at all oscillation frequencies; this bias is likely to erroneously affect the clinical interpretation of respiratory resistance and reactance if measurements obtained on the tremoFlo are interpreted using IOS-specific normative values; this applies particularly to patients with higher resistance. The findings suggest the need for better standardisation of measurements across oscillometry devices. Until then, it seems prudent to use instrument-specific normative values for interpretation.

The commercialisation of new oscillometry devices raises the question as to whether normative values elaborated on another device, using a different waveform, data algorithm and/or oscillation frequency, can be used interchangeably. The literature on the topic is scarce with only three publications; all identified measurement discrepancies, both in vitro and/or in vivo, between oscillometry devices $[15,16,22]$. We observed lower resistance values at $\geqslant 10 \mathrm{~Hz}$, higher frequency dependence of resistance $(\Delta R 5-20)$ and less 


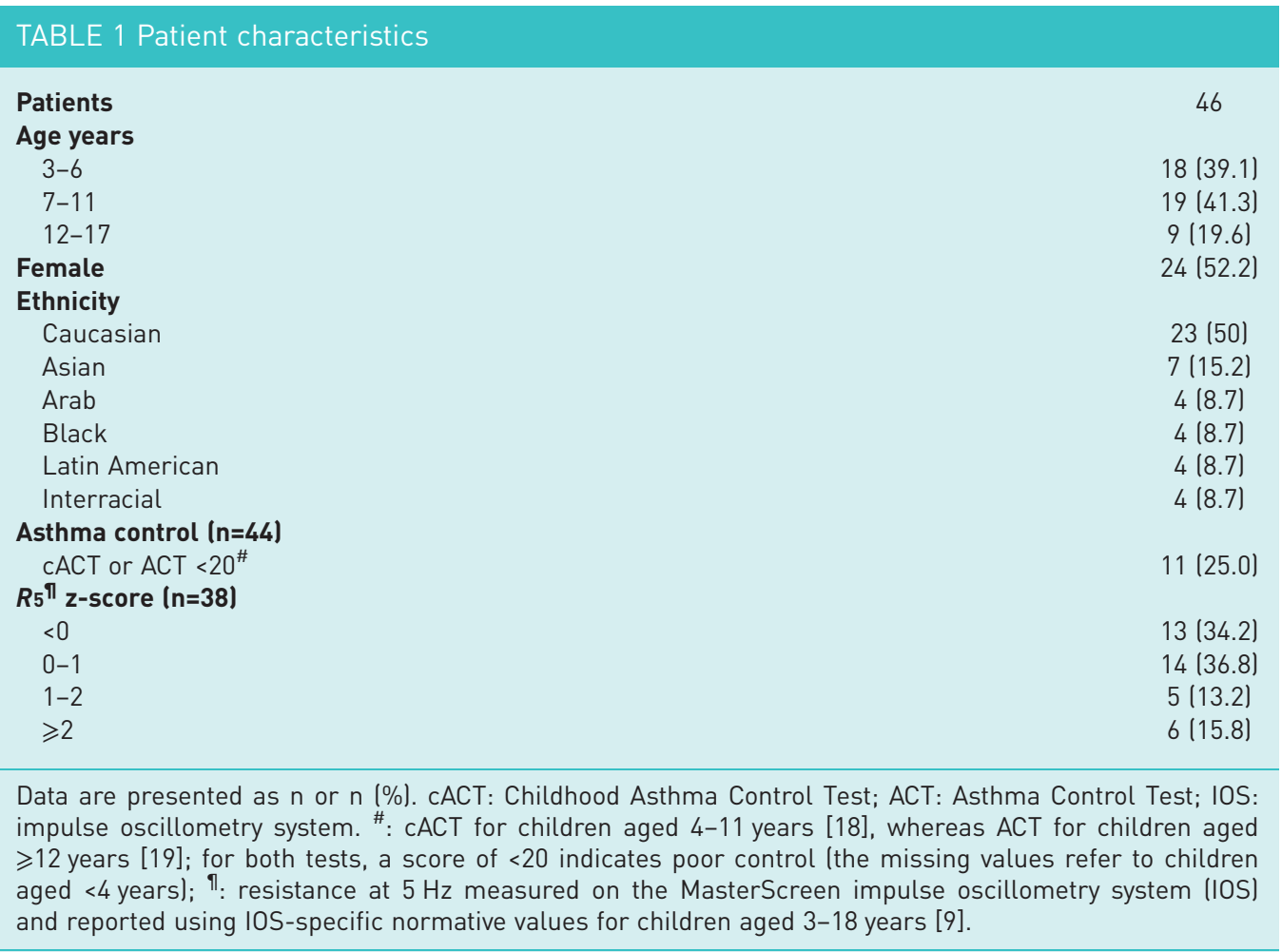

negative reactance on the IOS than the tremoFlo. These findings are consistent with the $10 \%$ difference observed by TANimura et al. [15] comparing the IOS to the MostGraph using test charges of 0.196, 0.402 and $0.951 \mathrm{kPa} \cdot \mathrm{L}^{-1} \cdot \mathrm{s}^{-1}$ under different respiratory rates and tidal volumes. Zimmermann et al. [16] compared the tremoFlo, Resmon Pro, IOS and a custom-built device ("WIMR") in 12 healthy adults as well as against a fixed resistance of $0.20 \mathrm{kPa} \cdot \mathrm{L}^{-1} \cdot \mathrm{s}^{-1}$ and reactance of $-0.31 \mathrm{kPa} \cdot \mathrm{L}^{-1} \cdot \mathrm{s}^{-1}$. Although no significant differences were observed between the test charges and devices, the in vivo resistance on the

TABLE 2 Within-patient absolute agreement between tremoFlo and MasterScreen impulse oscillometry system (IOS) values

\begin{tabular}{|c|c|c|c|c|c|c|}
\hline & \multirow[t]{2}{*}{ Patients n } & \multicolumn{2}{|c|}{ tremoFlo $\mathrm{kPa} \cdot \mathrm{L}^{-1} \cdot \mathrm{s}^{-1}$} & \multicolumn{2}{|c|}{$10 S \mathrm{kPa} \cdot \mathrm{L}^{-1} \cdot \mathrm{s}^{-1}$} & \multirow[t]{2}{*}{ tremoFlo versus IOS ICC $(95 \% \mathrm{CI})$} \\
\hline & & Mean士sD & Median (IQR) & Mean \pm SD & Median (IQR) & \\
\hline \multicolumn{7}{|c|}{ Resistance $^{\#}$} \\
\hline$R 10$ & 38 & $0.74 \pm 0.30$ & $0.67(0.28)$ & $0.66 \pm 0.21$ & $0.60(0.26)$ & $0.91(0.82-0.95)$ \\
\hline$R 15$ & 38 & $0.63 \pm 0.25$ & $0.57(0.25)$ & $0.60 \pm 0.17$ & $0.54(0.22)$ & $0.90(0.80-0.95)$ \\
\hline$R_{20}$ & 38 & $0.59 \pm 0.22$ & $0.53(0.26)$ & $0.56 \pm 0.15$ & $0.52(0.19)$ & $0.88(0.77-0.94)$ \\
\hline \multicolumn{7}{|c|}{ Reactance $^{\#}$} \\
\hline$x_{5}$ & 38 & $-0.36 \pm 0.20$ & $-0.31(0.16)$ & $-0.19 \pm 0.14$ & $-0.18(0.14)$ & $0.75(0.52-0.87)$ \\
\hline$X_{10}$ & 38 & $-0.18 \pm 0.17$ & $-0.13(0.16)$ & $-0.16 \pm 0.13$ & $-0.14(0.15)$ & $0.82(0.66-0.91)$ \\
\hline$X_{15}$ & 38 & $-0.17 \pm 0.16$ & $-0.12(0.12)$ & $-0.10 \pm 0.11$ & $-0.09(0.14)$ & $0.87(0.76-0.93)$ \\
\hline$X_{20}$ & 38 & $-0.09 \pm 0.14$ & $-0.06(0.11)$ & $-0.02 \pm 0.08$ & $-0.01(0.09)$ & $0.78(0.58-0.89)$ \\
\hline$X_{25}$ & 38 & $0.002 \pm 0.123$ & $0.02(0.12)$ & $0.06 \pm 0.06$ & $0.05(0.09)$ & $0.69(0.40-0.84)$ \\
\hline $\begin{array}{l}\text { IQR: in } \\
\text { represe } \\
\text { parame } \\
\text { patients }\end{array}$ & $\begin{array}{l}\text { range; IC } \\
\text { ice and } \lambda \\
\text { red at } 5-\end{array}$ & $\begin{array}{l}\text { ents rea } \\
\text { only ava }\end{array}$ & the trem & rea und & $\begin{array}{l}\text { urve for re } \\
\text { ncluding th } \\
\text { nent was ex }\end{array}$ & $\begin{array}{l}\text { e; Fres: resonant frequency. }{ }^{\#}: R \\
\text { ency dependency between } R \text { or } X \\
\text { with the IOS; }{ }^{+}: \text {reported only for }\end{array}$ \\
\hline
\end{tabular}



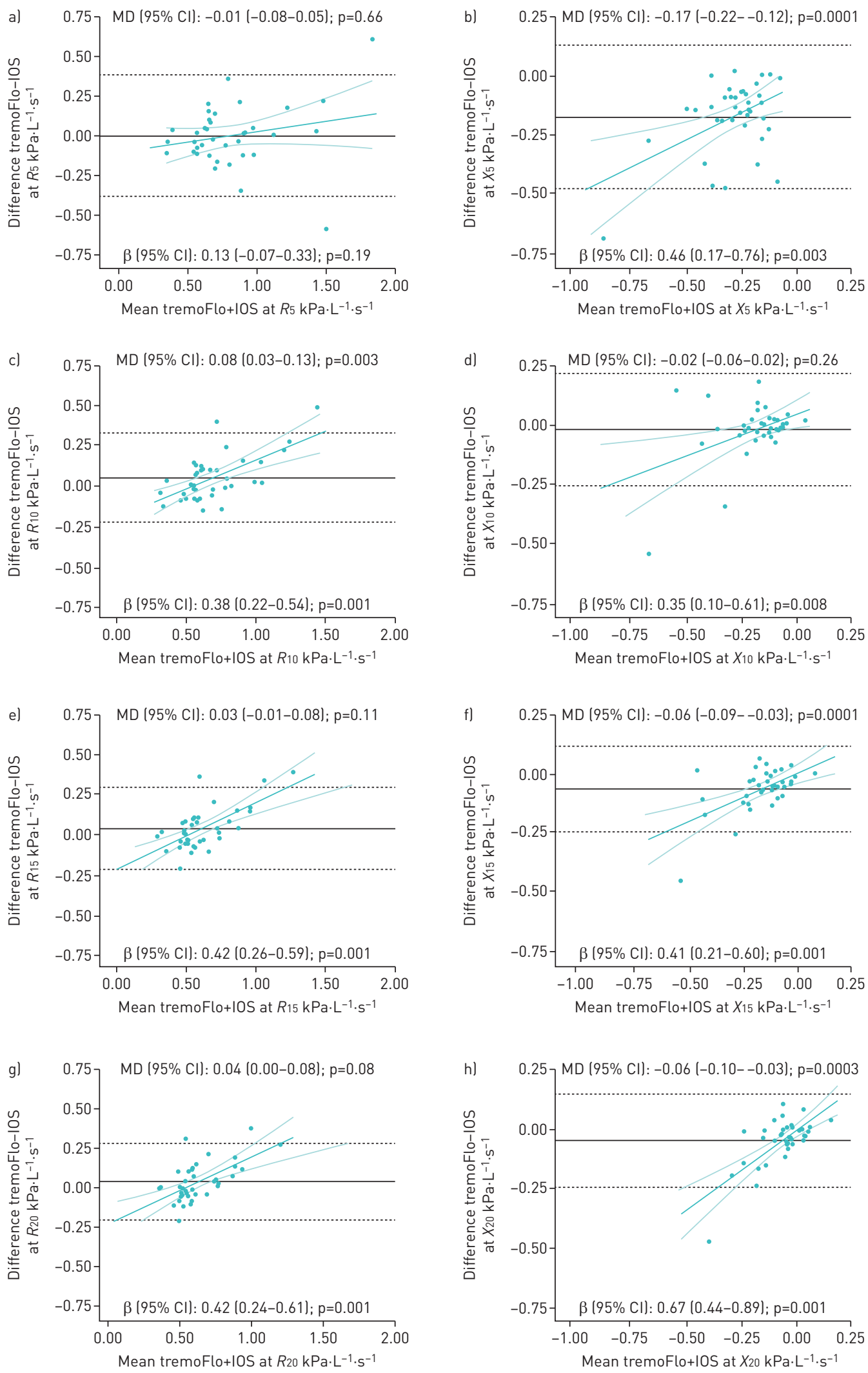

FIGURE 2 See next page for caption. 

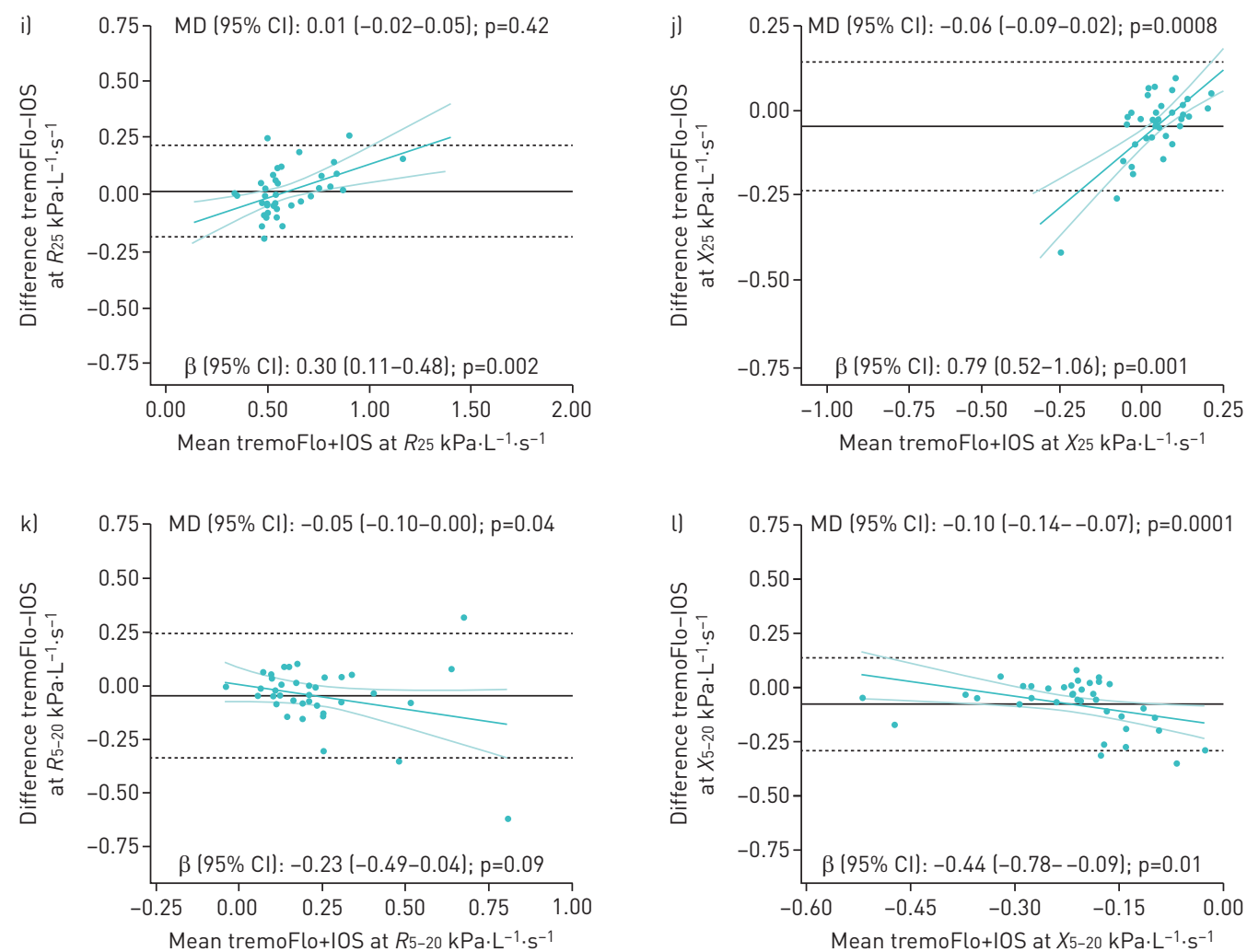

FIGURE 2 a-l) Bland-Altman plots of absolute differences between tremoFlo and MasterScreen impulse oscillometry system (IOS) measurements versus the mean of the two measurements in resistance $R$ (left panels) at a) 5, c) 10, e) 15, g) 20 , i) 25 and k) $5-20 \mathrm{~Hz}$ and in reactance $X$ (right panels) at b) 5, d) 10, f) 15 , h) 20 , j) 25 and () $5-20 \mathrm{~Hz}$ in 38 patients with reproducible IOS measurements. MD: mean difference. In each plot, the black solid horizontal line represents the absolute within-patient MD between parameters measured on the tremoFlo minus those on the IOS, with $95 \%$ of all absolute differences contained within the black dashed horizontal lines. These values are indicated as MD $(95 \% \mathrm{Cl})$ in $\mathrm{kPa} \cdot \mathrm{L}^{-1} \cdot \mathrm{s}^{-1}$ at the top of each plot: values $>0$ indicate that tremoFlo measurements were higher than IOS measurements and vice versa. The magnitude and direction of the proportional bias is depicted by the green linear regression line with the $95 \%$ confidence interval and is indicated as $B(95 \% \mathrm{Cl})$ at the bottom of each plot.

\section{TABLE 3 Projected impact of observed proportional bias on interpretation}

Resistance

Measurements

Value measured
on IOS

on IOS

$\mathrm{kPa} \cdot \mathrm{L}^{-1} \cdot \mathrm{s}^{-1}$

Projected value
measured on
tremoFlo"
$\mathrm{kPa} \cdot \mathrm{L}^{-1} \cdot \mathrm{s}^{-1}$

0.73
1.01
1.29
1.58
0.85
1.19
1.54
1.88

0.73

1

1.25

1.5

$R 10$

0.75

1

1.5
1.01

1.29

1.19

1.88 4-year-old male $(105 \mathrm{~cm})$

\begin{tabular}{ccc}
\hline IOS value & tremoFlo & Difference \\
z-score & value & z-score $^{+}$ \\
& z-score &
\end{tabular}

$-1.35$

$-0.27$

0.82

1.90

$-0.71$

0.39

1.50

2.61

-1.44
-0.22
1.01
2.24
-0.28
1.24
2.77
4.30

-1.44
-0.22

1.01

$-0.28$

1.24

4.30

0.09
-0.05
-0.19
-0.33
-0.43
-0.85
-1.27
-1.69

$-0.05$

$-0.19$

$-0.43$

$-0.85$

$-1.69$ 8.5-year-old male $(129 \mathrm{~cm})$

\begin{tabular}{|c|c|c|}
\hline $\begin{array}{l}\text { IOS value } \\
\text { z-score }\end{array}$ & $\begin{array}{l}\text { tremoFlo } \\
\text { value } \\
\text { z-score }\end{array}$ & $\begin{array}{c}\text { Difference } \\
\text { z-score }^{+}\end{array}$ \\
\hline
\end{tabular}

IOS: MasterScreen impulse oscillometry system. ${ }^{\#}$ : based on the observed average resistance- and oscillatory frequency-specific proportional bias (note: we conservatively used the bias observed in 38 children, without removing the outlier identified for R5); ${ }^{1}$ : z-score values calculated using IOS-specific paediatric normative values [9]; ${ }^{+}: \mathrm{z}$-score difference between those derived from the IOS versus tremoFlo, with negative values indicating a lower $z$-score value on the IOS than the tremoFlo. 

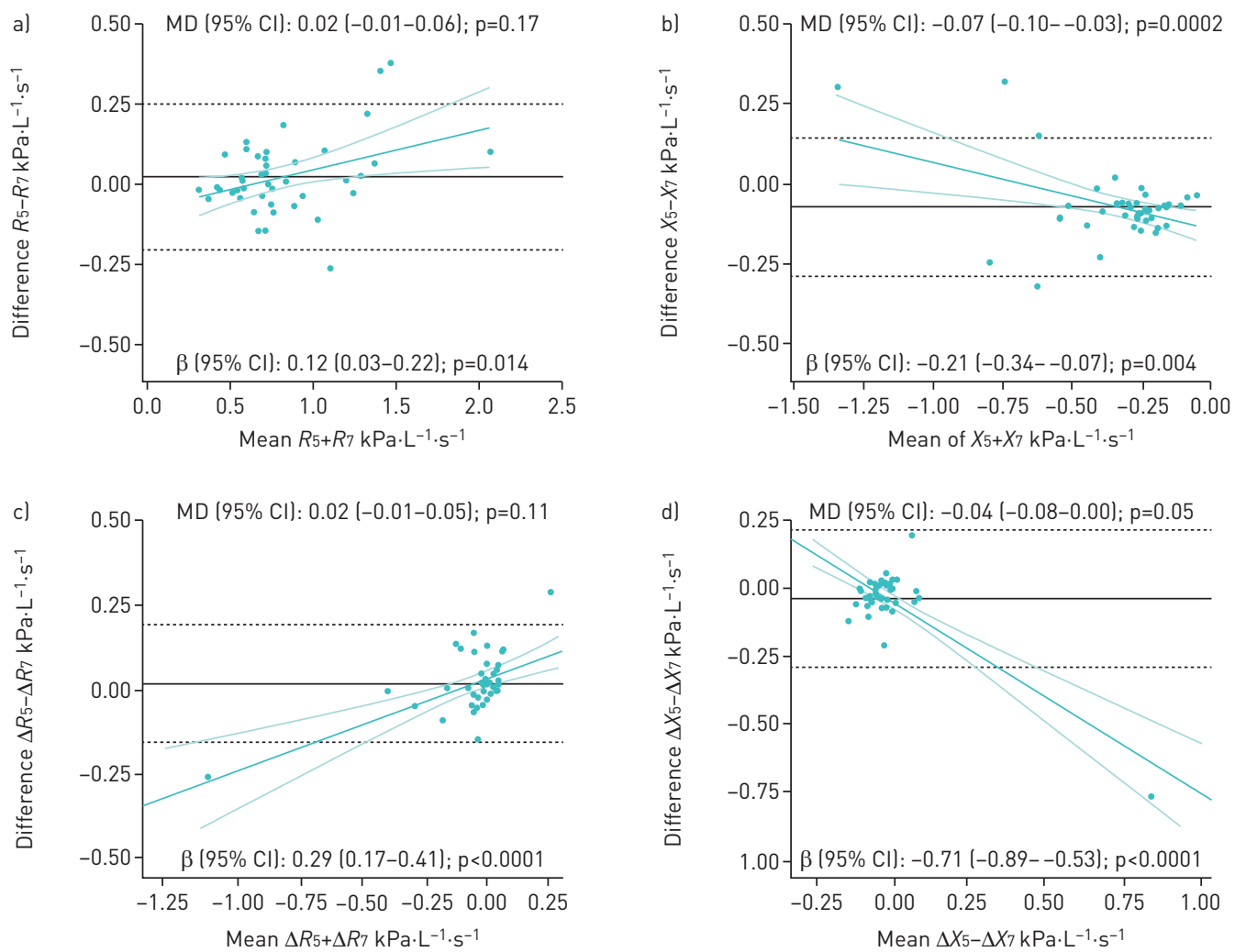

FIGURE 3 a-d) Bland-Altman plots of absolute differences between tremoFlo at 5 and $7 \mathrm{~Hz}$ versus the mean of the two measurements in resistance $R$ and reactance $X$ in 44 patients with reproducible measurements for a) $R$ and b) $X$ and in 42 patients for c) $\Delta R$ and d) $\Delta X$. MD: mean difference. In each plot, the black solid horizontal line represents the absolute MD, with $95 \%$ of all absolute differences contained within the black dashed horizontal lines. These values are indicated as $\mathrm{MD}(95 \% \mathrm{Cl})$ in $\mathrm{kPa} \cdot \mathrm{L}^{-1} \cdot \mathrm{s}^{-1}$ at the top of each plot: values $>0$ indicate that tremoFlo measurements at $5 \mathrm{~Hz}$ are higher than at $7 \mathrm{~Hz}$ and vice versa. The magnitude and direction of the proportional bias is depicted by the green linear regression line with the $95 \%$ confidence interval and is indicated as B $(95 \% \mathrm{Cl})$ at the bottom of each plot.

IOS differed significantly from that of the other two devices, with higher resistance and more negative reactance in the former. The direction of these differences contradicted our findings and is probably explained in part by the higher resistance displayed by our asthmatic children, with a mean $R 5$ of $0.80 \mathrm{kPa} \cdot \mathrm{L}^{-1} \cdot \mathrm{s}^{-1}$ compared with $<0.3 \mathrm{kPa} \cdot \mathrm{L}^{-1} \cdot \mathrm{s}^{-1}$ in healthy adults, and the test charges used; moreover, our larger spectrum of resistance from 0.30 to $1.78 \mathrm{kPa} \cdot \mathrm{L}^{-1} \cdot \mathrm{s}^{-1}$ on the IOS enabled the identification of a proportional bias, which was probably not detectable in healthy adults or with a low test charge. To date, other comparisons of IOS with other non-IOS instruments using test charges or subjects have only been published in abstract form [23-25]; they consistently reported significant between-device variation exceeding within-patient variability, particularly with parameters other than $R 5$, supporting between-device impedance differences.

Our observed between-device overall difference in resistance was negligible at $5 \mathrm{~Hz}$, one of the most commonly used frequencies. However, the post hoc exclusion of an outlier revealed a similar proportional bias to that observed with all other oscillatory frequencies; this observation deserves further confirmation. With increasing interest in reactance and in the frequency dependence of resistance as more sensitive markers of small airway obstruction than resistance at $5 \mathrm{~Hz}$, the observed between-device difference may be problematic. Importantly, the observed proportional bias in resistance at $\geqslant 10 \mathrm{~Hz}$ (and $5 \mathrm{~Hz}$, after removing an outlier) and in reactance at all oscillation frequencies increased by $\sim 30 \%$ for every $1 \mathrm{kPa} \cdot \mathrm{L}^{-1} \cdot \mathrm{s}^{-1}$. In our case simulations, the distortion between the values obtained on IOS versus tremoFlo was most clinically important above $1 \mathrm{kPa} \cdot \mathrm{L}^{-1} \cdot \mathrm{s}^{-1}$ for $R 10$, where a $\mathrm{z}$-score difference in the interpretation exceeded 0.80 . This suggests that the between-device difference may cause significant distortion in the interpretation of findings.

As expected, resistance values were marginally lower, but reactance values were significantly less negative at 7 than $5 \mathrm{~Hz}$ for all intrabreath reactance parameters (inspiratory, expiratory and $\Delta X$ ). Yet, the statistically significant proportional bias indicates that the interpretation of resistance or reactance at $7 \mathrm{~Hz}$ 
using $5 \mathrm{~Hz}$ normative values would underestimate the severity of obstruction, particularly in small children and more obstructed individuals. Frequency-specific reference values are thus required.

The study must be interpreted in light of the following strengths and limitations. Acknowledging various sources of biases to explain measurement discrepancies, including differences in the population, setting, measurement procedures, within-patient variation and device specifications, our design aimed to minimise the former four biases. We performed within-patient comparison of measurements obtained in the same setting, using the same procedures, with two operators blinded to measurements obtained on the alternate device, allocation concealment until randomisation and random testing order; the latter served to avoid systematic differences due to patient fatigue with repeated measurements. While we cannot exclude the possibility of within-patient between-measurement variation, such variation is reported to be small $[17,26]$, and would be random and thus minimised by the randomised measurement order. Observed between-device measurement differences are therefore more likely to be due to differences in device specifications, including transducers and data processing algorithms. Perhaps a higher within-patient variation in diseased than in healthy individuals could have contributed to the observed between-device variation [27]; however, the observations were made in asthmatic children, a group in whom oscillatory resistance is frequently used. We recognised the possibility of a lower accuracy in measuring $R 5$ from a $5-25 \mathrm{~Hz}$ signal than $R 7$ with a monofrequency oscillation. The adequate study power and consistency of findings across various agreement parameters underline the robustness of the findings. In the absence of correction for multiple testing, we cannot rule out the possibility of false-positive findings. As we enrolled asthmatic children with a large spectrum of impedance, a sizeable proportion of participants had elevated resistance, enabling the identification of a proportional bias of $\geqslant 30 \%$. Yet, given the small absolute number of children with elevated resistance ( $z$-score $\geqslant 2$ in six children), replication of our findings is needed. In the absence of explicit guideline recommendations regarding the best way to assess reproducibility [28], we excluded children with a coefficient of variation $>15 \%$ of resistance at $5 \mathrm{~Hz}$ (or $7 \mathrm{~Hz}$ ), resulting in a mean coefficient of variation of $8 \%$; perhaps longer measurements would have led to lower within-patient variability and fewer exclusions [29]. In the absence of a true gold standard, this comparative study was not designed to identify the oscillometry device providing the most accurate measurements, only to explore agreement and systematic differences. As for generalisation, as participants were predominantly asthmatic children, caution is advised before extrapolating results to adults and healthy children.

Although oscillometry may be more accessible and sensitive to detect small airway obstruction than spirometry [30, 31], the observed between-device impedance measurement differences represent an important obstacle to its widespread use. Whereas general recommendations have been issued regarding device specifications $[2,28]$, reference values for oscillometry still vary significantly, interfering with the pooling of normative data $[9,14,32]$. Clearly, it is imperative to understand and address between-device measurement discrepancies, including the observed fixed and proportional biases, across commercially available devices to facilitate standardisation of oscillometry measurements.

In conclusion, the excellent agreement, small absolute difference and apparent absence of systematic bias in resistance values measured at $5 \mathrm{~Hz}$ suggest that $R 5$ normative values developed on the IOS may perhaps be used for the tremoFlo (although caution is advised due to the impact of outlier removal). However, reference values at $5 \mathrm{~Hz}$ cannot be applied to $7 \mathrm{~Hz}$ measurements. Due to the large proportional bias observed, the use of tremoFlo-specific normative values is preferred for resistance at $\geqslant 10 \mathrm{~Hz}$, frequency dependence and all reactance parameters, to avoid significant discordance in the interpretation of airflow obstruction, particularly in patients with expected higher resistance, such as young children and in those with airflow obstruction.

Acknowledgements: We acknowledge the Fonds de la Recherche du Québec en Santé for the infrastructure support provided to the Research Centre of the CHU Sainte-Justine (Montreal, QC, Canada). The authors would like to thank Pei-Yin Liao and Zaynab Ridha (Research Centre, CHU Sainte-Justine) for their participation in patient recruitment, and Christine Lord (Research Centre, CHU Sainte-Justine) for assisting in the study coordination. We thank Thorasys for lending the tremoFlo device used in this study.

Author contributions: F.M. Ducharme developed the research question, supervised the conception of the research protocol and the statistical analysis, interpreted results, and wrote the final version manuscript. I. Jroundi assisted in developing the protocol, coordinated the study under supervision, initiated the statistical analysis and wrote the first draft of the manuscript. G. Lavoie Boutin and G. Jean drafted the first version of the protocol and conducted oscillatory testing. B. Vinet, I. Jroundi and C. Lawson conducted the statistical analyses.

Conflict of interest: F.M. Ducharme reports nonfinancial support (donation of the tremoFlo device) from Thorasys Inc. during the conduct of the study. I. Jroundi reports receiving a Canadian Francophonie Scholarship Program (CFSP) postdoctoral scholarship during the conduct of the study. G. Jean has nothing to disclose. G. Lavoie Boutin has nothing to disclose. C. Lawson has nothing to disclose. B. Vinet has nothing to disclose. 
Support statement: This project (2017-1552) was funded by the Academic Chair in Clinical and Knowledge Transfer Research on Childhood Asthma of the Sainte-Justine University Health Centre. We acknowledge the Programme Canadien de Bourses de la Francophonie/Canadian Francophonie Scholarship Program, funded by the Government of Canada, for the postdoctoral scholarship awarded to I. Jroundi. Funding information for this article has been deposited with the Crossref Funder Registry.

\section{References}

1 Beydon N. Pulmonary function testing in young children. Paediatr Respir Rev 2009; 10: 208-213.

2 Beydon N, Davis SD, Lombardi E, et al. An official American Thoracic Society/European Respiratory Society statement: pulmonary function testing in preschool children. Am J Respir Crit Care Med 2007; 175: 1304-1345.

3 Ducharme FM, Davis GM. Respiratory resistance in the emergency department: a reproducible and responsive measure of asthma severity. Chest 1998; 113: 1566-1572.

4 Rosenfeld M, Allen J, Arets BH, et al. An official American Thoracic Society workshop report: optimal lung function tests for monitoring cystic fibrosis, bronchopulmonary dysplasia, and recurrent wheezing in children less than 6 years of age. Ann Am Thorac Soc 2013; 10: S1-S11.

5 Debley J, Filbrun AG, Subbarao P. Clinical applications of pediatric pulmonary function testing: lung function in recurrent wheezing and asthma. Pediatr Allergy Immunol Pulmonol 2011; 24: 69-76.

6 Ducharme FM, Davis GM. Measurement of respiratory resistance in the emergency department: feasibility in young children with acute asthma. Chest 1997; 111: 1519-1525.

7 Frei J, Jutla J, Kramer G, et al. Impulse oscillometry: reference values in children 100 to $150 \mathrm{~cm}$ in height and 3 to 10 years of age. Chest 2005; 128: 1266-1273.

8 Dencker M, Malmberg LP, Valind S, et al. Reference values for respiratory system impedance by using impulse oscillometry in children aged 2-11 years. Clin Physiol Funct Imaging 2006; 6: 247-250.

9 Nowowiejska B, Tomalak W, Radliński J, et al. Transient reference values for impulse oscillometry for children aged 3-18 years. Pediatr Pulmonol 2008; 43: 1193-1197.

10 Malmberg LP, Pelkonen A, Poussa T, et al. Determinants of respiratory system input impedance and bronchodilator response in healthy Finnish preschool children. Clin Physiol Funct Imaging 2002; 22: 64-71.

11 Lee JY, Seo JH, Kim HY, et al. Reference values of impulse oscillometry and its utility in the diagnosis of asthma in young Korean children. J Asthma 2012; 49: 811-816.

12 Amra B, Soltaninejad F, Golshan M. Respiratory resistance by impulse oscillometry in healthy Iranian children aged 5-19 years. Iran J Allergy Asthma Immunol 2008; 7: 25-29.

13 Park JH, Yoon JW, Shin YH, et al. Reference values for respiratory system impedance using impulse oscillometry in healthy preschool children. Korean J Pediatr 2011; 54: 64-68.

14 AlBlooshi A, AlKalbani A, Narchi $\mathrm{H}$, et al. Respiratory function in healthy Emirati children using forced oscillations. Pediatr Pulmonol 2018; 53: 936-941.

15 Tanimura K, Hirai T, Sato S, et al. Comparison of two devices for respiratory impedance measurement using a forced oscillation technique: basic study using phantom models. J Physiol Sci 2014; 64: 377-382.

16 Zimmermann SC, Watts JC, Bertolin A, et al. Discrepancy between in vivo and in vitro comparisons of forced oscillation devices. J Clin Monit Comput 2018; 32: 509-512.

17 Hall GL, Sly PD, Fukushima T, et al. Respiratory function in healthy young children using forced oscillations. Thorax 2007; 62: 521-526.

18 Liu AH, Zeiger R, Sorkness C, et al. Development and cross-sectional validation of the Childhood Asthma Control Test. J Allergy Clin Immunol 2007; 119: 817-825.

19 Nathan RA, Sorkness CA, Kosinski M, et al. Development of the asthma control test: a survey for assessing asthma control. J Allergy Clin Immunol 2004; 113: 59-65.

20 Koo TK, Li MY. A guideline of selecting and reporting intraclass correlation coefficients for reliability research. J Chiropr Med 2016; 15: 155-163.

21 Bland JM, Altman DG. Statistical methods for assessing agreement between two methods of clinical measurement. Lancet 1986; 1: 307-310.

22 Hellinckx J, Cauberghs M, De Boeck K, et al. Evaluation of impulse oscillation system: comparison with forced oscillation technique and body plethysmography. Eur Respir J 2001; 18: 564-570.

23 Oostveen E, De Soomer K, Otte JA, et al. Instrument variability in the measurement of respiratory resistance. Eur Respir J 2014; 44: Suppl. 58, P1823.

24 Put W, Baars J, van der Grinten CPM. Comparing 3 forced oscillation devices in different subject groups. Eur Respir J 2016; 48: Suppl. 60, PA3613.

25 Sato S, Hirai T, Tanimura K, et al. Comparison of three devices for respiratory impedance measurement using forced oscillation technique: basic study using phantom models. Am J Respir Crit Care Med 2015; 191: A2100.

26 Zimmermann S, Lyon J, Bertolin A, et al. Within-breath resistance and reactance and short-term repeatability in two forced oscillation technique devices. Eur Respir J 2015; 46: Suppl. 59, PA2276.

27 Timmins SC, Coatsworth N, Palnitkar G, et al. Day-to-day variability of oscillatory impedance and spirometry in asthma and COPD. Respir Physiol Neurobiol 2013; 185: 416-424.

28 Oostveen E, MacLeod D, Lorino $\mathrm{H}$, et al. The forced oscillation technique in clinical practice: methodology, recommendations and future developments. Eur Respir J 2003; 22: 1026-1041.

29 Watts JC, Farah CS, Seccombe LM, et al. Measurement duration impacts variability but not impedance measured by the forced oscillation technique in healthy, asthma and COPD subjects. ERJ Open Res 2016; 2: 00094-02015.

30 Skylogianni E, Douros K, Anthracopoulos MB, et al. The forced oscillation technique in paediatric respiratory practice. Paediatr Respir Rev 2016; 18: 46-51.

31 Komarow HD, Myles IA, Uzzaman A, et al. Impulse oscillometry in the evaluation of diseases of the airways in children. Ann Allergy Asthma Immunol 2011; 106: 191-199.

32 Calogero C, Simpson SJ, Lombardi E, et al. Respiratory impedance and bronchodilator responsiveness in healthy children aged 2-13 years. Pediatr Pulmonol 2013; 48: 707-715. 